\title{
Valvotomia Mitral Percutânea: Experiência Inicial com o Novo Balão Único de Dilatação "Tipo Inoue"
}

\author{
Cristiano de Oliveira Cardoso ${ }^{1}$, Erlon Oliveira de Abreu Silva ${ }^{1}$, La Hore Corrêa Rodrigues Jr. ${ }^{1}$, \\ Sabrina Torrano ${ }^{1}$, Carlos Roberto Cardoso ${ }^{1}$, José Carlos Haertel ${ }^{1}$, Carlos Antônio Mascia Gottschall' \\ La Hore Corrêa Rodrigues ${ }^{1}$
}

\section{RESUMO}

Introdução: A valvotomia mitral percutânea com único balão é a técnica mais utilizada para tratamento de pacientes com estenose mitral sintomática. Este estudo teve como objetivo avaliar o desempenho do novo balão único de dilatação "tipo Inoue" em pacientes com estenose mitral sintomática submetidos a valvotomia mitral percutânea com único balão. Método: Participaram do estudo pacientes com estenose mitral sintomática submetidos a valvotomia mitral percutânea com único balão entre março de 2008 e setembro de 2009, comparando o grupo tratado com balão "tipo Inoue" ao grupo tratado com balão clássico de Inoue. Ecocardiograma antes e após a valvotomia mitral percutânea com único balão e seguimento clínico tardio foram realizados. Resultados: Os pacientes foram divididos em dois grupos de comparação: grupo A, novo balão $(n=16)$; e grupo $B$, balão de Inoue $(n=10)$. Ambos os grupos apresentavam características clínicas semelhantes. A área mitral aumentou de 1,03 $\pm 0,24 \mathrm{~cm}^{2}$ para 1,75 \pm $0,22 \mathrm{~cm}^{2}$ no grupo $\mathrm{A}$, e de $1,08 \pm 0,25 \mathrm{~cm}^{2}$ para $1,88 \pm$ $0,41 \mathrm{~cm}^{2}$ no grupo $B(P=0,33)$. Não ocorreram casos de perfuração cardíaca, tamponamento ou insuficiência mitral grave requerendo cirurgia. Duas pacientes apresentaram hematoma $>10 \mathrm{~cm}$ no grupo $\mathrm{A}(12,5 \%$ no grupo $\mathrm{A}$ vs. 0 no grupo $B ; P=0,27)$. A sobrevivência livre de eventos foi de $100 \%$ no grupo $A$ e de $90 \%$ no grupo $B(P=0,21)$. Conclusões: A valvotomia mitral percutânea por balão mostrou-se segura e eficaz. Neste estudo, o novo balão de dilatação demonstrou resultados superponíveis aos do balão clássico de Inoue.

DESCRITORES: Estenose da valva mitral. Dilatação com balão. Cateterismo cardíaco. Ecocardiografia.

\footnotetext{
1 Instituto de Cardiologia do Rio Grande do Sul - Fundação Universitária de Cardiologia (IC-FUC) - Porto Alegre, RS, Brasil. Correspondência: Cristiano de Oliveira Cardoso. Rua Francisco Petuco, 340/805 - Boa Vista - Porto Alegre, RS, Brasil - CEP 90520-620 E-mail: cristiano.cardoso@cardiologia.org.br
}

Recebido em: 7/9/2009 • Aceito em: 21/11/2009

\section{ABSTRACT}

Percutaneous Mitral Commissurotomy: Initial Experience with the New Inoue-Type Balloon

Background: Percutaneous mitral commissurotomy (PMC) with a single balloon is the most used technique to treat symptomatic mitral stenosis. The objective of this study was to evaluate a new single balloon (Inoue-type) in patients with symptomatic mitral stenosis undergoing percutaneous mitral commissurotomy with a single balloon. Method: Patients undergoing PMC with a single balloon from March/2008 to September/2009 were included, comparing the group treated with the Inoue-type balloon to the group treated with Inoue's classic balloon. Pre and post procedure echocardiogram and late follow up were carried out. Results: Patients were divided into two groups for comparison: A, Inoue-type balloon $(n=16)$ and $B$, original Inoue balloon $(\mathrm{n}=10)$. Both groups presented similar clinical characteristics. Mitral valve area increased from $1.03 \pm 0.24 \mathrm{~cm}^{2}$ to $1.75 \pm 0.22 \mathrm{~cm}^{2}$ in group $\mathrm{A}$ and $1.08 \pm$ $0.25 \mathrm{~cm}^{2}$ to $1.88 \pm 0.41 \mathrm{~cm}^{2}$ in $\mathrm{B}(\mathrm{P}=0.33)$. There were no cases of cardiac perforation, cardiac tamponate and severe mitral insufficiency requiring surgery. Two group $A$ patients presented hematoma $>10 \mathrm{~cm}(12.5 \%$ in group $A$ vs. 0 in group $B ; P=0.27$ ). Event free survival was $100 \%$ in group $A$ and $90 \%$ in group $B(P=0.21)$. Conclusions: Percutaneous mitral commissurotomy is safe and effective. The new Inoue-type balloon showed comparable results with Inoue's classic balloon.

DESCRIPTORS: Mitral valve stenosis. Balloon dilatation Heart catheterization. Echocardiography.

E mbora a prevalência de febre reumática tenha sido significativamente reduzida nos dias atuais, casos de estenose mitral decorrente de comprometimento reumatismal ainda são encontrados na prática cardiológica diária'. De acordo com as diretrizes vigentes, pacientes sintomáticos com área valvar mitral inferior a $1,5 \mathrm{~cm}^{2}$ e aparelho valvar preservado têm indicação classe I de tratamento com valvotomia mitral percutânea por balão ${ }^{2,3}$. 
Cardoso CO, et al. Valvotomia Mitral Percutânea: Experiência Inicial com o Novo Balão Único de Dilatação "Tipo Inoue". Rev Bras Cardiol Invas. 2009;17(4):518-25.

Desde a introdução da valvotomia mitral percutânea com Inoue, em 1984, várias técnicas de dilatação têm sido descritas. Apesar do avanço tecnológico constante na área cardiológica, a valvotomia mitral percutânea com único balão pela técnica de Inoue é a mais utilizada em comparação à técnica de duplo balão sobre duas guias ${ }^{5}$, à técnica de duplo balão sobre uma guia ${ }^{6,7}$ (sistema Multi-track) e ao dispositivo de Cribier ${ }^{8,9}$. Atualmente existem balões com desenho similar ao do balão original de Inoue, porém resultados específicos de cada modelo têm sido pouco relatados.

O objetivo do presente estudo é avaliar o desempenho do novo balão único de dilatação "tipo Inoue" em pacientes submetidos a valvotomia mitral percutânea por balão com estenose mitral sintomática.

\section{MÉTODO}

\section{Seleção da amostra}

Foram selecionados todos os pacientes submetidos a valvotomia mitral percutânea com único balão entre março de 2008 e setembro de 2009 no Instituto de Cardiologia do Rio Grande do Sul (Porto Alegre, RS). Todos os pacientes tratados apresentavam quadro clínico de insuficiência cardíaca classe funcional II-IV pela New York Heart Association (NYHA) e estenose mitral moderada a grave (área mitral $<1,5 \mathrm{~cm}^{2}$ ).

\section{Avaliação clínica pré-procedimento}

Os pacientes foram avaliados clinicamente por meio de anamnese, exame físico e eletrocardiograma. Ecocardiograma transtorácico foi realizado em todos os pacientes, sendo a gravidade da estenose mitral mensurada pela área mitral (planimetria) e pelos gradientes máximo e médio ao Doppler. Também foram avaliados, por ecocardiograma, o aparelho valvar mitral pelo escore de Wilkins e Block ${ }^{10}$ e a presença de valvopatias associadas. Pacientes com ritmo cardíaco de fibrilação e/ou flutter atrial foram submetidos, adicionalmente, a ecocardiograma transesofágico para exclusão de possíveis trombos em átrio esquerdo. Para a realização das valvotomias mitrais percutâneas com único balão, foram utilizados apenas o novo balão testado (grupo A) e o balão clássico de Inoue (grupo B).

\section{Descrição do balão}

O novo balão único de dilatação "tipo Inoue" (Shenzhen Shineyard Medical Device Co., Ltd. Shenzhen, China) tem desenho semelhante ao do balão clássico de Inoue (Toray - Tóquio, Japão). Trata-se de um balão de corpo único em polivinil, que, quando preenchido por contraste, apresenta dilatação não uniforme. Primeiramente ocorre insuflação da porção distal do balão (mais complacente) e à medida que é mantida a injeção de meio de contraste em seu interior a porção proximal do balão é dilatada (Figura 1A). A porção média do balão ("cintura") determina seu

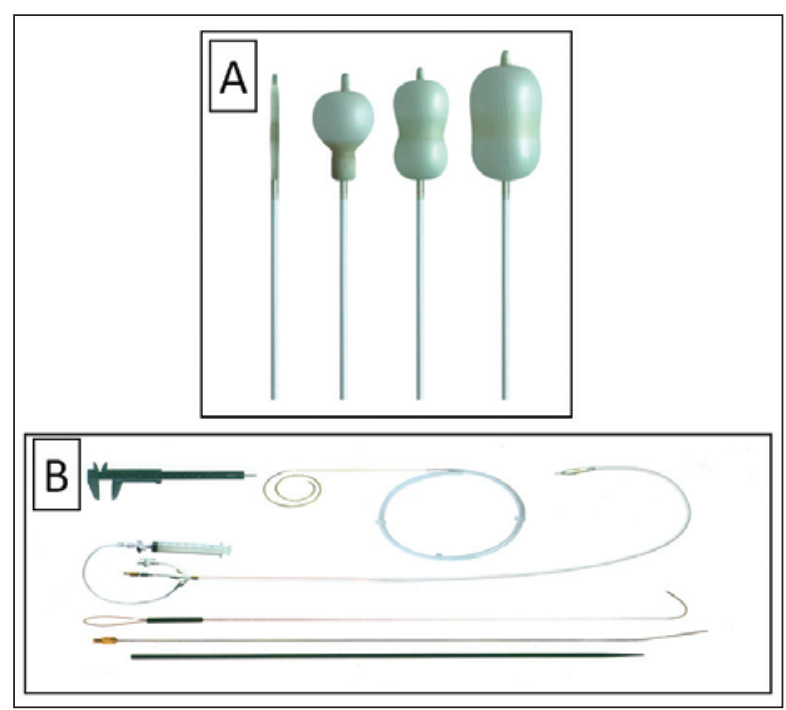

Figura 1 - Em A, novo balão "tipo Inoue" sendo progressivamente insuflado, observando-se que a porção distal infla antes que a proximal. Em B, os itens que acompanham o conjunto: guia espiralada, dilatador, estirador, direcionador, seringa e paquímetro.

diâmetro, e os tamanhos disponíveis variam de $20 \mathrm{~mm}$ a $30 \mathrm{~mm}$. O conjunto de dilatação é composto por guia com ponta espiralada, dilatador, estirador, direcionador, seringa e paquímetro (Figura 1B). O balão testado é comercializado no Brasil e está inscrito na Agência Nacional de Vigilância Sanitária (Anvisa) sob registro de número 80102510259 (www.anvisa.gov.br).

\section{Procedimento percutâneo}

Os procedimentos foram realizados no laboratório de hemodinâmica sob anestesia local e sedação. O procedimento percutâneo é descrito, sumariamente, a seguir: por via venosa femoral direita, foram cateterizadas e registradas as pressões nas câmaras cardíacas direitas. Por via arterial femoral esquerda registraram-se as pressões em aorta e ventrículo esquerdo, e marcou-se o plano valvar aórtico com cateter de pigtail. A seguir, posicionou-se a bainha de Mullins na altura do septo interatrial, puncionando-o com agulha de Brockenbrough em projeção oblíqua anterior esquerda 45 graus. Após confirmação da posição adequada em átrio esquerdo, dilatou-se o septo interatrial com dilatador $14 \mathrm{~F}$ e avaliou-se o gradiente transmitral. Heparinizouse o paciente com $5.000 \mathrm{U}$ de heparina por via venosa periférica. Utilizando o balão de valvotomia, dilatouse a válvula mitral progessivamente até redução significativa do gradiente transmitral. O procedimento foi finalizado com novo registro pressórico em câmaras cardíacas direita e esquerda, ventriculografia esquerda em projeção oblíqua anterior direita 35 graus para avaliação do plano valvar mitral e retirada dos introdutores valvulados. O cálculo para seleção do tamanho do balão foi baseado na seguinte fórmula ${ }^{11}$ : [(altura em cm $\div$ 10) + 10] 


\section{Seguimento clínico}

Os pacientes realizaram ecocardiograma transtorácico no dia seguinte ao procedimento para avaliação do resultado. Avaliação clínica funcional foi realizada por meio de consulta médica regular, revisão de prontuário ou contato telefônico.

\section{Definições}

Neste estudo foram adotadas as seguintes definições:

1) sucesso - área mitral pós-dilatação superior a $1,5 \mathrm{~cm}^{2}$ na ausência de regurgitação mitral grave ou outra complicação;

2) sucesso parcial ou resultado subótimo - área mitral $<1,5 \mathrm{~cm}^{2}$ na ausência de regurgitação mitral grave ou outra complicação;

3) insucesso - impossibilidade de dilatação valvar ou falha na punção do septo interatrial;

4) complicações maiores - morte, perfuração cardíaca com ou sem tamponamento, acidente vascular encefálico relacionado ao procedimento, hematoma retroperitoneal e insuficiência mitral grave requerendo cirurgia;

5) complicações menores - hematoma no sítio de punção femoral $>10 \mathrm{~cm}$ de diâmetro, fístula arteriovenosa, arritmia relacionada ao procedimento e pseudoaneurisma femoral; e

6) sobrevivência livre de eventos - sobrevivência livre de óbito, cirurgia de troca valvar mitral, nova valvoplastia mitral ou mudança do grau funcional pela NYHA.

\section{Análise estatística}

Os resultados são apresentados em média, desvio padrão e porcentual. Para comparação das variáveis categóricas foi utilizado o teste de qui-quadrado, sendo as variáveis contínuas analisadas pelos testes $t$ de Student, quando distribuídas normalmente, e nãoparamétrico, no caso de distribuição não normal. Sobrevivência livre de eventos foi mensurada pela curva de Kaplan-Meier, com teste de Log Rank. Foi considerado nível de significância estatística valor de $\mathrm{P}$ bicaudal inferior a 0,05. Os dados foram avaliados na versão 11.0 do Programa SPSS para Windows.

\section{RESULTADOS}

No período do estudo, foram realizadas 27 valvotomias mitrais percutâneas com único balão. Em um caso não foi possível a punção do septo interatrial (falha global de punção de 3,7\%). Tratava-se de uma paciente com comissurotomia cirúrgica prévia com acesso via toracotomia lateral direita. Nesse tipo de abordagem ocorre incisão do septo interatrial e poste- rior sutura. O processo de cicatrização por fibrose leva ao espessamento do septo interatrial, que dificulta sua punção.

Nos 26 pacientes restantes foi possível realizar o procedimento de dilatação valvar, sendo 16 pacientes incluídos no grupo A (novo balão) e 10, no grupo B (balão de Inoue).

A Tabela 1 apresenta as características clínicas dos pacientes incluídos no estudo e a Tabela 2 mostra os achados eletrocardiográficos e ecocardiográficos pré-procedimento. Não foram observadas diferenças entre os grupos, exceto maior número de pacientes com insuficiência cardíaca classe IV e menor complexidade ecocardiográfica do aparelho valvar mitral pelo escore de Wilkins e Block no grupo B.

\section{Resultados imediatos}

O sucesso do procedimento foi de 100\% (16/16) no grupo $A$ e de $90 \%(9 / 10)$ no grupo $B(P=0,85)$. $\mathrm{O}$ único paciente em quem não se obteve área mitral $>1,5 \mathrm{~cm}^{2}$ no grupo $\mathrm{B}$ foi um caso com resultado subótimo. A paciente apresentou área mitral final de $1,45 \mathrm{~cm}^{2}$, inferior a $1,5 \mathrm{~cm}^{2}$, definida previamente como critério de sucesso.

Conseguiu-se aumento da área valvar mitral de $69 \%$ no grupo A e de $74 \%$ no grupo B. Na Figura 2 estão demonstrados os resultados do incremento significativo da área mitral após valvotomia por balão de $1,03 \pm 0,24 \mathrm{~cm}^{2}$ para $1,75 \pm 0,22 \mathrm{~cm}^{2}$ no grupo A e de 1,08 $\pm 0,25 \mathrm{~cm}^{2}$ para $1,88 \pm 0,41 \mathrm{~cm}^{2}$ no grupo $B(P=0,33)$.

Os resultados da manometria em ambos os grupos são apresentados na Tabela 3. As pressões sistólica e diastólica na artéria pulmonar, que têm íntima relação com a gravidade da estenose mitral, sofreram alterações agudas pós-valvotomia mitral. O gradiente transmitral também foi reduzido, após o procedimento, de $16,1 \pm 6,2 \mathrm{mmHg}$ para $5,9 \pm 2,9 \mathrm{mmHg}$ no grupo A e de 18,9 $\pm 9,1 \mathrm{mmHg}$ para 5,2 $\pm 4,2 \mathrm{mmHg}$ no grupo $B(P=0,68)$.

\section{Resultados tardios}

O seguimento tardio, realizado em todos os pacientes, revela boa evolução clínica em ambos os grupos. A sobrevivência livre de eventos, avaliada pela curva de Kaplan-Meier, não demonstrou diferença estatística entre os grupos (Figura 3). Apenas uma paciente do grupo B apresentou retorno dos sintomas de insuficiência cardíaca pouco mais de quatro meses após a valvotomia mitral percutânea com único balão, com piora do grau de regurgitação pós-procedimento (evoluiu de regurgitação mitral leve para moderada), compensada com o uso de betabloqueador, diurético e inibidor da enzima de conversão. A paciente mantém-se assintomática até o presente momento. 
Cardoso CO, et al. Valvotomia Mitral Percutânea: Experiência Inicial com o Novo Balão Único de Dilatação "Tipo Inoue". Rev Bras Cardiol Invas. 2009;17(4):518-25.

Tabela 1

Características clínicas dos pacientes tratados com valvotomia mitral percutânea por balão

\begin{tabular}{|c|c|c|c|}
\hline Características clínicas, n (\%) & Grupo A $(n=16)$ & Grupo B $(n=10)$ & $\mathbf{P}$ \\
\hline Sexo feminino & $7 / 16(43,75 \%)$ & $8 / 10(80 \%)$ & 0,35 \\
\hline Idade (anos) & $43,68 \pm 14,55$ & $37,7 \pm 12,97$ & 0,48 \\
\hline Peso (kg) & $67,86 \pm 16,49$ & $64,9 \pm 9,48$ & 0,61 \\
\hline Altura $(\mathrm{cm})$ & $162 \pm 7,44$ & $158,7 \pm 8,02$ & 0,26 \\
\hline Hipertensão arterial sistêmica & $6 / 16(37,5 \%)$ & $2 / 10(20 \%)$ & 0,48 \\
\hline Diabetes melito & 0 & $2 / 10(20 \%)$ & 0,09 \\
\hline Dislipidemia & $4 / 16(25 \%)$ & $2 / 10(20 \%)$ & 0,81 \\
\hline Tabagismo & $2 / 16(12,5 \%)$ & $1 / 10(10 \%)$ & 0,86 \\
\hline AVC prévio & $1 / 6(6,25 \%)$ & $0 / 10(0 \%)$ & 0,43 \\
\hline Plastia cirúrgica prévia & $4 / 16(25 \%)$ & $0 / 10(0 \%)$ & 0,12 \\
\hline Plastia percutânea prévia & 0 & 0 & $\mathrm{~N} / \mathrm{A}$ \\
\hline Insuficiência cardíaca & & & 0,01 \\
\hline Classe II (NYHA) & $3 / 16(18,75 \%)$ & $2 / 10(20 \%)$ & \\
\hline Classe III (NYHA) & $13 / 16(81,25)$ & $6 / 10(60 \%)$ & \\
\hline Classe IV (NYHA) & 0 & $2 / 10(20 \%)$ & \\
\hline \multicolumn{4}{|l|}{ Medicações em uso, n (\%) } \\
\hline Anticoagulante oral & $4 / 16(25 \%)$ & $2 / 10(20 \%)$ & 0,81 \\
\hline Ácido acetilsalicílico & $6 / 16(37,5 \%)$ & $4 / 10(40 \%)$ & 0,93 \\
\hline Betabloqueador & $12 / 16(75 \%)$ & $6 / 10(60 \%)$ & 0,36 \\
\hline Inibidor da enzima conversora da angiotensina & $7 / 16(43,75 \%)$ & $3 / 10(30 \%)$ & 0,63 \\
\hline Diurético & $12 / 16(75 \%)$ & $6 / 10(60 \%)$ & 0,72 \\
\hline Inibidor dos receptores da angiotensina & $1 / 16(6,25 \%)$ & $1 / 10(10 \%)$ & 0,74 \\
\hline Digoxina & $4 / 16(25 \%)$ & $1 / 10(10 \%)$ & 0,42 \\
\hline
\end{tabular}

Resultados apresentados em média, desvio padrão e porcentual.

AVC = acidente vascular cerebral; $\mathrm{n}=$ número de pacientes; $\mathrm{N} / \mathrm{A}=$ não se aplica; NYHA = New York Heart Association.

\section{Complicações}

Não ocorreram complicações maiores (morte, perfuração cardíaca com ou sem tamponamento, acidente vascular encefálico relacionado ao procedimento, hematoma retroperitoneal e insuficiência mitral grave requerendo cirurgia) nos dois grupos.

Entre as complicações definidas como menores, não foi observada presença de fístula arteriovenosa, arritmia relacionada ao procedimento e pseudoaneurisma femoral. Ocorreram dois casos de hematoma relacionado ao sítio de punção $>10 \mathrm{~cm}$ de diâmetro no grupo A $(12,5 \%$ no grupo A vs. 0 no grupo B; $P=0,27)$. Ambas as pacientes eram portadoras de fibrilação atrial crônica e, por decisão do cardiologista assistente, iniciaram o uso de anticoagulante oral cerca de 24 horas após a retirada dos introdutores valvulados. Os hematomas foram tratados clinicamente e resolvidos espontaneamente.

A piora do grau de regurgitação mitral é uma das principais complicações da valvotomia mitral percutânea com único balão. Neste estudo, observou-se diferença entre os grupos em relação à piora da regurgitação mitral pós-procedimento (0 no grupo A vs. 3 pacientes no grupo $B ; P=0,04)$. Esses pacientes do grupo $B$ apresentaram piora do grau de regurgitação mitral, de leve para moderada, e a avaliação com ecocardiograma identificou ruptura parcial de cordoalha tendínea em um e nenhuma complicação mecânica nos demais. Além disso, esses pacientes demonstraram, ao final do procedimento, áreas mitrais significantemente maiores que aqueles que não evoluíram com essa complicação $\left(1,07 \pm 0,24 \mathrm{~cm}^{2}\right.$ para 2,12 $\pm 0,48 \mathrm{~cm}^{2}$ vs. 1,05 \pm $0,23 \mathrm{~cm}^{2}$ para $\left.1,73 \pm 0,21 \mathrm{~cm}^{2} ; \mathrm{P}=0,004\right)$. No grupo A, foi observada uma regressão no grau de regurgitação mitral após valvotomia mitral percutânea com único balão. Tratava-se de uma paciente com estenose mitral grave (área de $0,88 \mathrm{~cm}^{2}$ ) e significativa restrição da mobilidade do folheto anterior, que melhorou sua função após dilatação valvar.

\section{Valvotomia mitral percutânea pós-comissurotomia cirúrgica}

Comissurotomia cirúrgica prévia estava presente em 4 pacientes $(15 \%$ da amostra), sendo todos tratados percutaneamente com o novo balão "tipo Inoue" 
Tabela 2

Achados eletrocardiográficos e ecocardiográficos pré-procedimento nos pacientes tratados com valvotomia mitral percutânea

\begin{tabular}{|c|c|c|c|}
\hline & Grupo A $(n=16)$ & Grupo B (n = 10) & $\mathbf{P}$ \\
\hline \multicolumn{4}{|l|}{ Achados ecocardiográficos, n (\%) } \\
\hline Área mitral (cm²) & $1,03 \pm 0,24$ & $1,08 \pm 0,25$ & 0,62 \\
\hline Gradiente transmitral máximo $(\mathrm{mmHg})$ & $20,29 \pm 7,10$ & $21,91 \pm 6,17$ & 0,58 \\
\hline Gradiente transmitral médio $(\mathrm{mmHg})$ & $10,59 \pm 3,93$ & $12,3 \pm 2,84$ & 0,27 \\
\hline Escore de Wilkins e Block & & & $<0,001$ \\
\hline$<8$ & $15 / 16(93,75 \%)$ & $10 / 10(100 \%)$ & \\
\hline $9-11$ & $1 / 16(6,25 \%)$ & 0 & \\
\hline$>12$ & 0 & 0 & \\
\hline Átrio esquerdo (mm) & $50,61 \pm 4,35$ & $48,37 \pm 2,77$ & 0,21 \\
\hline Fração de ejeção (\%) & $65,42 \pm 7,2$ & $64,42 \pm 4,42$ & 0,65 \\
\hline Trombo atrial esquerdo (\%) & 0 & 0 & $\mathrm{~N} / \mathrm{A}$ \\
\hline \multicolumn{4}{|l|}{ Valvulopatia associada, n (\%) } \\
\hline Estenose aórtica leve & $2 / 16(12,5 \%)$ & $4 / 10(40 \%)$ & 0,20 \\
\hline Estenose aórtica moderada a grave & 0 & 0 & $\mathrm{~N} / \mathrm{A}$ \\
\hline Insuficiência aórtica leve & $3 / 16(18,75 \%)$ & 9/10 (90\%) & 0,03 \\
\hline Insuficiência aórtica moderada a grave & 0 & 0 & N/A \\
\hline Insuficiência mitral leve & $13 / 16(81,25 \%)$ & 9/10 (90\%) & 0,86 \\
\hline Insuficiência mitral moderada a grave & $1 / 16(6,25 \%)$ & 0 & 0,43 \\
\hline \multicolumn{4}{|l|}{ Achados eletrocardiográficos, n (\%) } \\
\hline Ritmo sinusal & $11 / 16(68,75 \%)$ & $8 / 10(80 \%)$ & 0,80 \\
\hline Fibrilação ou flutter atrial & $5 / 16(31,25 \%)$ & $2 / 10(20 \%)$ & 0,69 \\
\hline Sobrecarga atrial esquerda & $11 / 16(68,75 \%)$ & $8 / 10(80 \%)$ & 0,80 \\
\hline Sobrecarga ventricular esquerda & $4 / 16(25 \%)$ & $4 / 10(40 \%)$ & 0,56 \\
\hline Sobrecarga ventricular direita & $6 / 16(37,5 \%)$ & $1 / 10(10 \%)$ & 0,22 \\
\hline
\end{tabular}

Resultados apresentados em média, desvio padrão e porcentual.

$\mathrm{n}=$ número de pacientes; N/A = não se aplica.

(25\% no grupo A vs. 0 no grupo $B ; P=0,21)$. Nesse subgrupo $(100 \%$ dos pacientes com escore de Wilkins e Block inferior a 8) ocorreu aumento da área mitral $\left(1,03 \pm 0,28 \mathrm{~cm}^{2}\right.$ para 1,77 $\left.\pm 0,24 \mathrm{~cm}^{2}\right)$, sem ser observada piora do grau de regurgitação mitral pósprocedimento.

\section{DISCUSSÃO}

O presente estudo procurou avaliar o desempenho de dois balões com desenho e método de dilatação valvar semelhantes. Ambos os balões foram altamente eficazes no aumento da área valvar mitral após sua dilatação, com número reduzido de complicações. O procedimento de valvotomia mitral percutânea com único balão é realizado universalmente e seus resultados são amplamente conhecidos ${ }^{2,12}$. Em pacientes com estenose mitral moderada a grave com aparelho valvar preservado, o procedimento de valvotomia mitral percutânea com único balão tem indicação classe । sobre o procedimento cirúrgico ${ }^{2,13}$, em decorrência dos resultados extremamente favoráveis.
Apesar de existirem inúmeras técnicas de dilatação, a valvotomia com único balão ainda é a mais utilizada. A técnica de balão único tem algumas vantagens em relação aos outros métodos de dilatação ${ }^{14}$ : apresenta menor grau de dificuldade, pois a manipulação do cateter é mais fácil e seu uso não necessita guia; o risco de perfuração cardíaca também é menor; e, embora se obtenha área menor que a de duplo balão, a evolução clínica tardia de ambos os métodos é semelhante ${ }^{15,16}$. Neste estudo, o novo balão de dilatação demonstrou resultados superponíveis aos do balão clássico de Inoue, evidenciados pela similar taxa de sucesso e pelo baixo índice de complicações.

Insuficiência mitral grave pós-valvotomia mitral percutânea com único balão é uma das principais complicações. Tem sido reportada em 1,4\% a 9,4\% $\%^{7,17,18}$ dos procedimentos, embora casos com necessidade de cirurgia imediata sejam ainda mais raros. Nesta série, não houve registro de casos de regurgitação mitral grave com necessidade de cirurgia, ocorrendo apenas piora do grau de regurgitação mitral de leve para moderada em 
Cardoso CO, et al. Valvotomia Mitral Percutânea: Experiência Inicial com o Novo Balão Único de Dilatação "Tipo Inoue". Rev Bras Cardiol Invas. 2009;17(4):518-25.

3 pacientes tratados com balão de Inoue. A piora do grau de regurgitação mitral é relativamente frequente pós-valvotomia mitral percutânea com único balão ${ }^{19,20}$. Seu aparecimento está relacionado a vários fatores, como grau de calcificação mitral, comprometimento do apareIho subvalvar e área final alcançada. Como os pacientes que apresentaram essa complicação mostrassem escore ecocardiográfico adequado (Wilkins e Block < 8), acredita-se que esse achado tenha decorrido predominantemente do aumento significativo da área mitral média nesse grupo. A média de área final nesses pacientes foi de $2,12 \mathrm{~cm}^{2}$ e áreas valvares superiores têm sido relacionados a essa complicação ${ }^{19,20}$. Apesar disso, a evolução clínica é geralmente favorável e o tratamento clínico mantém compensado esse grupo de pacientes.

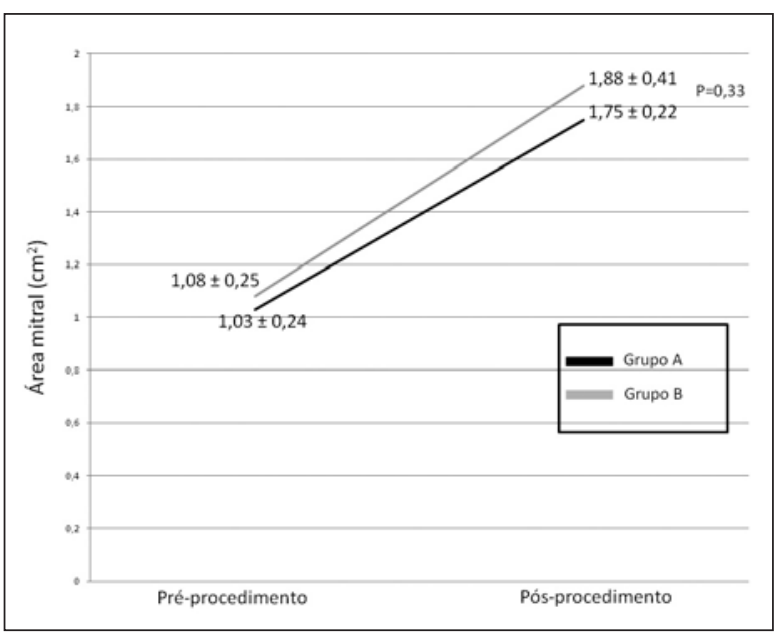

Figura 2 - Área mitral pré e pós-procedimento nos grupos submetidos a valvotomia mitral percutânea por balão.
A evolução natural dos pacientes tratados tanto por comissurotomia cirúrgica como por valvotomia mitral percutânea com único balão demonstra que cerca de $11 \%$ a $39 \%$ irão apresentar reestenose clínica e ecocardiográfica 7 a 15 anos após o procedimento $^{21}$. A realização de nova intervenção, geralmente, é indispensável, pelo retorno de sintomas. Em pacientes tratados cirurgicamente e com baixo escore ecocardiográfico, a valvotomia mitral percutânea com único balão pode ser realizada com segurança ${ }^{22-27}$. Estudos prévios demonstram a eficácia e a segurança desse procedimento, que se torna atrativo por evitar nova intervenção não-cirúrgica. Pacientes tratados por valvotomia mitral percutânea com único balão também podem ter seus procedimentos valvulares repetidos pela mesma

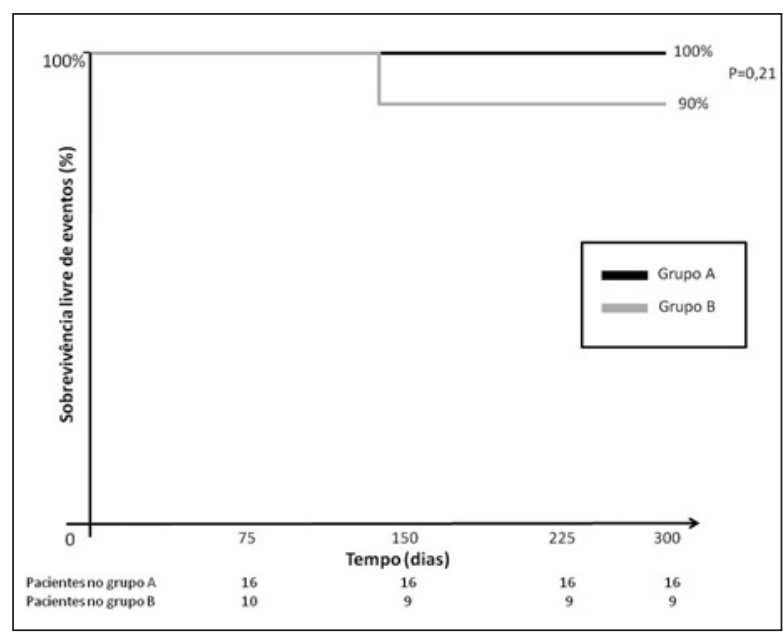

Figura 3 - Curva de sobrevivência livre de eventos estimada por Kaplan-Meier.

Tabela 3

Manometria das câmaras cardíacas antes e após valvotomia mitral percutânea

\begin{tabular}{lccc}
\hline & Grupo A (n= 16) & Grupo B (n= 10) & P \\
\hline Pré-procedimento (mmHg) & & & \\
Pressão sistólica em artéria pulmonar & $52,33 \pm 14,37$ & $50,95 \pm 13,15$ & 0,80 \\
Pressão diastólica em artéria pulmonar & $28,53 \pm 8,56$ & $28,7 \pm 17,60$ & 0,97 \\
Pressão sistólica em átrio esquerdo & $36,56 \pm 7$ & $36,85 \pm 6,80$ & 0,92 \\
Pressão diastólica em átrio esquerdo & $24,6 \pm 8,12$ & $19,3 \pm 7,08$ & 0,10 \\
Gradiente transmitral & $16,1 \pm 6,2$ & $18,9 \pm 9,1$ & 0,37 \\
Pós-procedimento (mmHg) & & & 0,83 \\
Pressão sistólica em artéria pulmonar & $45,75 \pm 14$ & $22,6 \pm 4,86$ & 0,87 \\
Pressão diastólica em artéria pulmonar & $23 \pm 6,72$ & $22,85 \pm 5,99$ & 0,35 \\
Pressão sistólica em átrio esquerdo & $26,64 \pm 8,58$ & $12,1 \pm 4,27$ & 0,008 \\
Pressão diastólica em átrio esquerdo & $19,6 \pm 7,05$ & $5,2 \pm 4,2$ & 0,68 \\
Gradiente transmitral & $5,9 \pm 2,9$ & & \\
\hline
\end{tabular}

Resultados apresentados em média e desvio padrão.

$\mathrm{n}=$ número de pacientes. 
técnica. Gomes et al. revelaram que esse tipo de procedimento é seguro pela técnica de balão, mesmo quando repetidos pela segunda e pela terceira vez ${ }^{28}$. No entanto, tende-se a obter áreas valvares menores.

\section{Seguimento em 12 meses}

O critério de sucesso da valvotomia mitral percutânea é definido como área valvar superior a 1,5 $\mathrm{cm}^{2}$. Embora essa área seja inferior à área normal da valva mitral $\left(3 \mathrm{~cm}^{2}\right.$ a $\left.5 \mathrm{~cm}^{2}\right)$, áreas superiores a $1,5 \mathrm{~cm}^{2}$ são suficientes para deixar o paciente livre de sintomas ${ }^{29,30}$. Esses achados também foram confirmados neste estudo, por meio da sobrevivência livre de eventos. Não houve diferença entre os grupos observada pela curva de Kaplan-Meier, o que sugere que ambos os balões tenham tido desempenho similar.

\section{Limitações do estudo}

As limitações pertinentes ao estudo são abordadas a seguir: 1) trata-se de estudo realizado em centro único, com número reduzido de pacientes; 2) a falta de randomização impede determinar a superioridade de um balão em relação ao outro; 3) embora a curva de sobrevida livre de eventos não demonstre diferença entre os grupos de tratamento, a ausência de controle ecocardiográfico tardio limita qualquer conclusão sobre a manutenção a longo prazo do resultado em relação à área mitral; e 4) em decorrência do pequeno número de pacientes incluídos, não foi possível determinar preditores de desfechos imediatos e tardios.

\section{CONCLUSÕES}

A valvotomia mitral percutânea por balão é um procedimento seguro e eficaz para o tratamento da estenose mitral moderada a grave sintomática. Neste estudo, o novo balão de dilatação testado demonstrou resultados superponíveis aos do balão clássico de Inoue.

\section{CONFLITO DE INTERESSES}

Os autores declararam inexistência de conflito de interesses relacionado a este manuscrito.

\section{REFERÊNCIAS BIBLIOGRÁFICAS}

1. Sociedade Brasileira de Cardiologia. Sociedade Brasileira de Pediatria. Sociedade Brasileira de Reumatologia. Diretrizes Brasileiras para o Diagnóstico, Tratamento e Prevenção da Febre Reumática. Arq Bras Cardiol [Internet]. 2009 [citado 2009 Nov 21];93(3 Supl 4):1-18. Disponível em: http:// publicacoes.cardiol.br/consenso/2009/diretriz febrereumatica 93supl04.pdf

2. Bonow RO, Carabello BA, Chatterjee $\mathrm{K}$, de Leon AC, Jr, Faxon DP, Freed MD, Gaasch WH, Llytle BW, Nishimura RA, O'Gara PT, O'Rourke RA, Otto CM, Shah PM, Shanewise JS; 2006 Writing Committee Members; American College of Cardiology/American Heart Association Task Force. 2008 Focused update incorporated into the ACC/AHA 2006 guidelines for the management of patients with valvular heart disease: a report of the American College of Cardiology/ American Heart Association Task Force on Practice Guidelines (Writing Committee to Revise the 1998 Guidelines for the Management of Patients With Valvular Heart Disease): endorsed by the Society of Cardiovascular Anesthesiologists, Society for Cardiovascular Angiography and Interventions, and Society of Thoracic Surgeons. Circulation. 2008;118(15):e523-661.

3. Pomerantzeff PM, Barbosa GV, de Sousa Filho BS, Brandao $C M$, Ribeiro EJ, Costa FD, et al. [Guidelines for surgery in heart valve diseases]. Arq Bras Cardiol. 2004;82 Suppl 5:2233. Portuguese.

4. Inoue K, Owaki T, Nakamura T, Kitamura F, Miyamoto N Clinical application of transvenous mitral commissurotomy by a new balloon catheter. J Thorac Cardiovasc Surg. 1984; 87(3):394-402.

5. Al Zaibag M, Ribeiro PA, Al Kasab S, Al Fagih MR. Percutaneous double-balloon mitral valvotomy for rheumatic mitral-valve stenosis. Lancet. 1986;1(8484):757-61.

6. lung B, Garbarz E, Michaud P, Helou S, Farah B, Berdah P, et al. Late results of percutaneous mitral commissurotomy in a series of 1024 patients. Analysis of late clinical deterioration: frequency, anatomic findings, and predictive factors. Circulation. 1999;99(25):3272-8. Comment in: Circulation. 2000;102(2):E18

7. Vahanian A, Michel PL, Cormier B, Vitoux B, Michel X, Slama $M$, et al. Results of percutaneous mitral commissurotomy in 200 patients. Am J Cardiol. 1989;63(12):847-52.

8. Cribier A, Rath PC, Letac B. Percutaneous mitral valvotomy with a metal dilatator. Lancet. 1997;349(9066):1667. Comment in: Lancet. 1997;350(9076):524.

9. Cribier A, Eltchaninoff $\mathrm{H}$, Koning $\mathrm{R}$, Rath $\mathrm{PC}$, Arora R, Imam $A$, et al. Percutaneous mechanical mitral commissurotomy with a newly designed metallic valvulotome: immediate results of the initial experience in 153 patients. Circulation. 1999; 99(6):793-9.

10. Wilkins GT, Weyman AE, Abascal VM, Block PC, Palacios IF. Percutaneous balloon dilatation of the mitral valve: an analysis of echocardiographic variables related to outcome and the mechanism of dilatation. Br Heart J. 1988;60(4):299-308.

11. Lau KW, Hung JS. A simple balloon-sizing method in Inoueballoon percutaneous transvenous mitral commissurotomy. Cathet Cardiovasc Diagn. 1994;33(2):120-9; discussion 1301. Comment in: Cathet Cardiovasc Diagn. 1995;35(2):183.

12. Dean LS, Mickel M, Bonan R, Holmes DR Jr, O'Neill WW, Palacios IF, et al. Four-year follow-up of patients undergoing percutaneous balloon mitral commissurotomy. A report from the National Heart, Lung, and Blood Institute Balloon Valvuloplasty Registry. J Am Coll Cardiol. 1996;28(6):1452-7.

13. Reyes VP, Raju BS, Wynne J, Stephenson LW, Raju R, Fromm BS, et al. Percutaneous balloon valvuloplasty compared with open surgical commissurotomy for mitral stenosis. N Engl J Med. 1994;331(15):961-7. Comment in: N Engl J Med. 1994;331(15):1014-5. N Engl J Med. 1995;332(11):748-9; author reply 749-50. N Engl J Med. 1995;332(11):748; author reply 749-50. N Engl J Med. 1995;332(11):749; author reply 749-50.

14. Fawzy ME, Mimish L, Sivanandam V, Lingamanaicker J, alAmri M, Khan B, et al. Advantage of Inoue balloon catheter in mitral balloon valvotomy: experience with 220 consecutive patients. Cathet Cardiovasc Diagn. 1996;38(1):9-14. Comment in: Cathet Cardiovasc Diagn. 1996;38(1):15.

15. Kang DH, Park SW, Song JK, Kim HS, Hong MK, Kim JJ, et al. Long-term clinical and echocardiographic outcome of percutaneous mitral valvuloplasty: randomized comparison of Inoue and double-balloon techniques. J Am Coll Cardiol. 2000;35(1):169-75.

16. Rifaie O, Abdel-Dayem MK, Ramzy A, Ezz-El-Din H, El- 
Cardoso CO, et al. Valvotomia Mitral Percutânea: Experiência Inicial com o Novo Balão Único de Dilatação "Tipo Inoue". Rev Bras Cardiol Invas. 2009;17(4):518-25.

Ziady G, El-Itriby A, et al. Percutaneous mitral valvotomy versus closed surgical commissurotomy. Up to 15 years of follow-up of a prospective randomized study. J Cardiol. 2009; 53(1):28-34

17. Palacios IF, Sanchez PL, Harrell LC, Weyman AE, Block PC. Which patients benefit from percutaneous mitral balloon valvuloplasty? Prevalvuloplasty and postvalvuloplasty variables that predict long-term outcome. Circulation. 2002;105(12): 1465-71.

18. Chen CR, Cheng TO. Percutaneous balloon mitral valvuloplasty by the Inoue technique: a multicenter study of 4832 patients in China. Am Heart J. 1995;129(6):1197-203.

19. Padial LR, Freitas N, Sagie A, Newell JB, Weyman AE, Levine RA, et al. Echocardiography can predict which patients will develop severe mitral regurgitation after percutaneous mitral valvulotomy. J Am Coll Cardiol. 1996;27(5):1225-31.

20. Padial LR, Abascal VM, Moreno PR, Weyman AE, Levine RA, Palacios IF. Echocardiography can predict the development of severe mitral regurgitation after percutaneous mitral valvuloplasty by the Inoue technique. Am J Cardiol. 1999;83(8):1210-3.

21. Fawzy ME. Percutaneous mitral balloon valvotomy. Catheter Cardiovasc Interv. 2007;69(2):313-21.

22. Medina A, Suarez De Lezo J, Hernandez E, Pan M, Romero M, Melian F, et al. Balloon valvuloplasty for mitral restenosis after previous surgery: a comparative study. Am Heart J. 1990; 120(3):568-71.

23. Serra $A$, Bonan $R$, Lefèvre $T$, Barraud $P$, Le Feuvre $C$, Leclerc $\mathrm{Y}$, et al. Balloon mitral commissurotomy for mitral restenosis after surgical commissurotomy. Am J Cardiol. 1993;71(15): $1311-5$.
24. Jang IK, Block PC, Newell JB, Tuzcu EM, Palacios IF. Percutaneous mitral balloon valvotomy for recurrent mitral stenosis after surgical commissurotomy. Am J Cardiol. 1995; 75(8):601-5.

25. Lau KW, Ding ZP, Gao W, Koh TH, Johan A. Percutaneous balloon mitral valvuloplasty in patients with mitral restenosis after previous surgical commissurotomy. A matched comparative study. Eur Heart J. 1996;17(9):1367-72.

26. Iung B, Garbarz E, Michaud P, Mahdhaoui A, Helou S, Farah B, et al. Percutaneous mitral commissurotomy for restenosis after surgical commissurotomy: late efficacy and implications for patient selection. J Am Coll Cardiol. 2000; 35(5):1295-302.

27. Fawzy ME, Hassan W, Shoukri M, Al Sanei A, Hamadanchi A, El Dali A, et al. Immediate and long-term results of mitral balloon valvotomy for restenosis following previous surgical or balloon mitral commissurotomy. Am J Cardiol. 2005 ; 96(7):971-5.

28. Gomes NL, Esteves VBC, Braga SLN, Ramos AIO, Esteves FA, Paes AT, et al. Valvotomia mitral percutânea: da primeira à terceira dilatação. Rev Bras Cardiol Invasiva. 2009;17(2):169-75.

29. Fawzy ME, Shoukri M, Fadel B, Badr A, Al Ghamdi A, Canver C. Long-term (up to 18 years) clinical and echocardiographic results of mitral balloon valvuloplasty in 531 consecutive patients and predictors of outcome. Cardiology. 2009;113(3):213-21.

30. Gomes NL, Esteves CA, Braga SL, Maldonado M, Machado L, Meneghelo ZM. Evolução tardia da valvoplastia mitral. Rev Soc Cardiol Estado de São Paulo. 2002;12(2):315-26. 